\title{
Probing the QSO host galaxy evolution through the gas metallicity
}

\author{
B. Husemann ${ }^{1}$, L. Wisotzki ${ }^{1}$, K. Jahnke ${ }^{2}$, S. F. Sánchez ${ }^{3}$, \\ and D. Nugroho ${ }^{2}$ \\ ${ }^{1}$ Leibniz-Institut für Astrophysik Potsdam, Germany \\ email: bhusemann@aip.de \\ ${ }^{2}$ Max-Planck-Institut für Astronomie, Heidelberg, Germany \\ ${ }^{3}$ Instituto de Astrofísica de Andalucía (CSIC), Granada, Spain
}

\begin{abstract}
We use the spatially resolved gas-phase metallicity as a new diagnostic for tagging recent interactions in QSO host galaxies. With this technique we also identified a QSO with extremely low gas-phase metallicity as likely evidence for gas accretion from the environment.
\end{abstract}

Keywords. galaxies: active, quasars: emission lines, HII regions, ISM: abundances

We measured the oxygen abundance distribution of QSO host galaxies at $z<0.2$ using integral-field spectroscopy. We find that the abundance gradients of disc-dominated QSO hosts are consistent with normal galaxies, but are flat or non-linear in bulge-dominated systems and ongoing mergers. The oxygen abundances at $1 R_{\mathrm{e}}$ are systematically lower in bulge-dominated QSO hosts as shown in Fig. 1. We interpret this as indication for recent (minor) interactions to trigger AGN in bulge-dominated hosts considering the expected metallicity dilution and systematic change in the gradients during merger events.

In Husemann et al. (2011), we presented the discovery of an extraordinary low gasphase metallicity in the NLR of the QSO HE 2158-0107 based on IFS observations. The [OIII] line brightness distribution reveals a $30 \mathrm{kpc}$ long tail-like structure of gas. Both facts are likely evidence for the accretion of nearly pristine gas from the environment either through minor merger(s) or smooth accretion of gas.

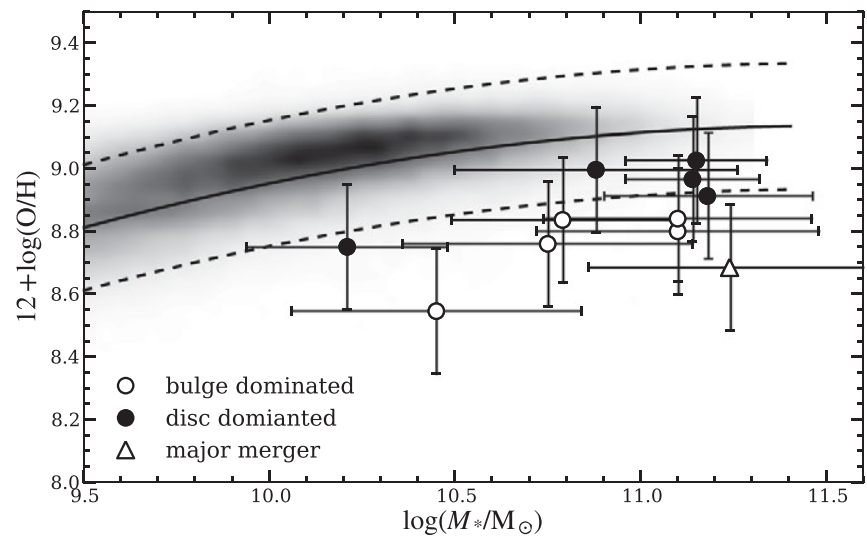

Figure 1. Estimated oxygen abundances at $1 R_{\mathrm{e}}$ for 11 QSO hosts against their total stellar mass. The global SDSS mass-metallicity relation of galaxies is shown for comparison.

\section{Reference}

Husemann, B., et al. 2011, A\&SA, 535, A72 